\title{
CONCEPTUAL DESIGN OF A CAPTURE RF SYSTEM FOR MUON COLLIDERS *
}

\author{
J. Rose \\ Brookhaven Lab, Upton, NY 11973, USA
}

\section{Abstract}

A conceptual RF System design provides the basis for a more detailed engineering study to explore the technical issues involved in fabricating and testing a capture RF system in a proton-driver target experiment. A large bore $71 \mathrm{MHz}$ cavity design is detailed which is self-consistent with a proton-driver target experiment at BNL. Analysis of cell to cell coupling in a linac composed of a string of such cavities is presented.

\section{INTRODUCTION}

Muon Colliders and Neutrino factories require proton driver accelerators that create pions from protons impinging upon fixed targets. The capture systems downstream from the target must collect the pion bunches which in longitudinal phase space are $\sim$ as short as the proton bunches that create them but have $\Delta \mathrm{E} / \mathrm{E}$ of $80 \%$ or more. One method of matching bunches into the subsequent acceleration channel is to perform a phase rotation in a low frequency RF system. A schematic of such a system is shown in figure 1 . The conceptual design of a target experiment on an AGS beam line at Brookhaven Lab was performed with RF cavities within a focusing solenoid [1]. Beam dynamics studies used a frequency of $60 \mathrm{MHz}$ and an accelerating gradient of $8 \mathrm{MV} / \mathrm{m}[2]$.

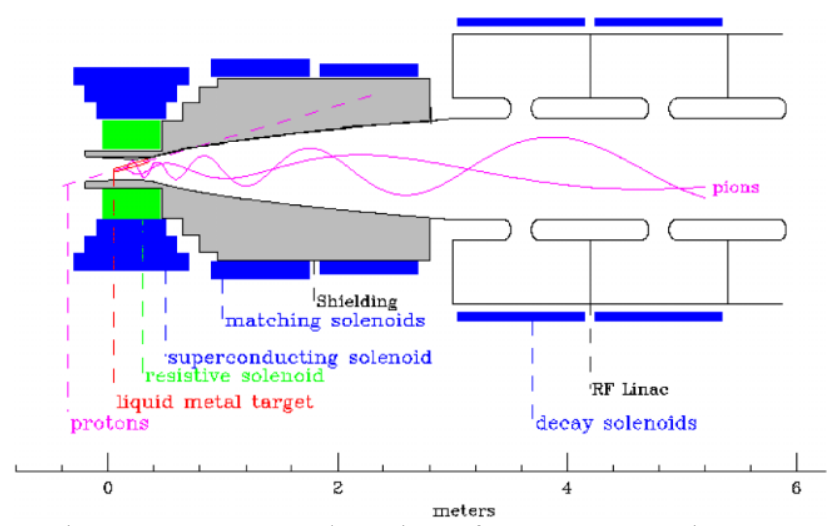

Figure 1. Conceptual Design of Capture Experiment

\section{SYSTEM DESIGN}

Based upon preliminary magnet design it was decided to incorporate the magnets into the RF cavity beam-tubes, bringing them much closer to the axis and therefore reducing the current and magnet mass required [3]. The goal for the RF input power per cavity was decided to be 5.5 MW based upon the availability from LBL of four
8973 tetrode amplifiers [4]. These are capable of 1.5MW peak power, with 1.5 millisecond pulse, $2 \mathrm{~Hz}$ at $\sim 70 \mathrm{MHz}$ which when combined could deliver up to $6 \mathrm{MW}$. Based on this input a self-consistent preliminary design was performed for $\sim 70 \mathrm{MHz}$ cavity for a target experiment at Brookhaven' AGS.

In order to be compatible with the g-2 experiment operations at the AGS from which we could take occasional AGS cycles and extract beam we need to phase lock the RF cavity to the AGS flat top frequency. The AGS parameters for g-2 operation are given in Table 1 .

Table 1: AGS operation for g-2

\begin{tabular}{|l|l|}
\hline Energy & $24 \mathrm{GeV}$ \\
\hline RF Harmonic & 12 \\
\hline RF Frequency & 4.45406 \\
\hline Revolution Freq & $371.17 \mathrm{kHz}$ \\
\hline
\end{tabular}

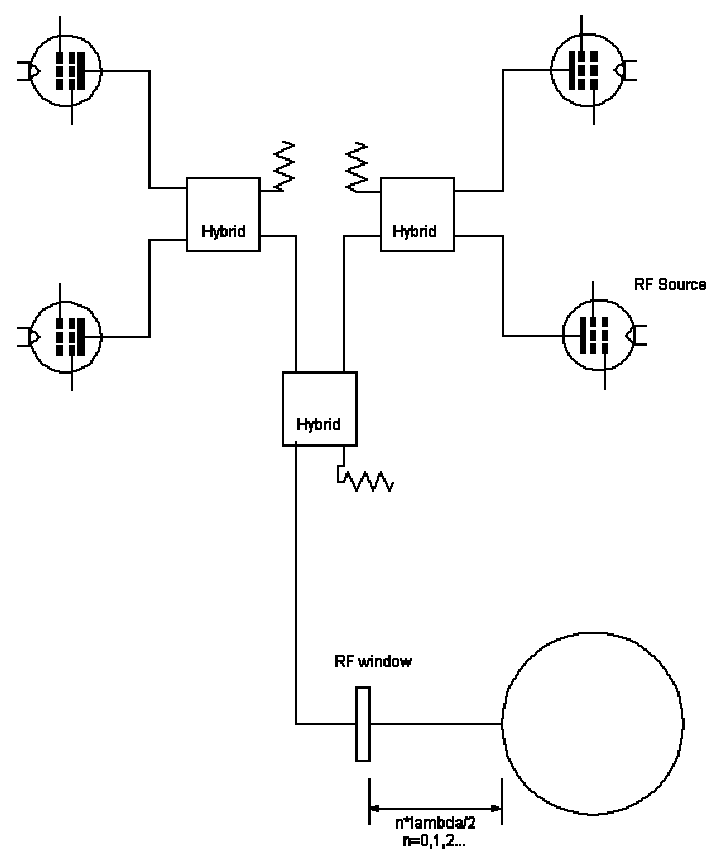

Figure 2. RF System layout showing power tetrodes, combining hybrids and window location for cavity.

In order to extract a train of 12 bunches synchronous to the target experiment cavity the cavity must be a harmonic of the AGS frequency. The closest harmonic to the $70 \mathrm{MHz}$ frequency of the LBL surplus Bevatron 8973 tetrodes to be used for the experiment is $16 \times 12=$

*Work supported by the Department of Energy. 
71.265MHz. If we give up on 12 bunches and instead go with 6 bunches we can use $31 \times 6=69.038 \mathrm{MHz}$. This is not much easier on the power amplifiers and eliminates the possibility of operating parasitically with g-2 operation. Therefore the design adopted the $71.26 \mathrm{MHz}$ for the frequency. Locking the cavity to the AGS flat top frequency is simple but necessitates an active tuner in the cavity.

\section{CAVITY DESIGN}

The cavity design started with a simple re-entrant cavity profile with the drift tubes sized to house the required magnets and a $2 \mathrm{~cm}$ buffer zone. The bore tube is $300 \mathrm{~mm}$ in radius and extends up to $470 \mathrm{~mm}$ and houses a magnet of half length $280 \mathrm{~mm}$. The system layout is shown in Figure 1. Preliminary cavity design parameters are given in Table 1.

Table $1.71 .26 \mathrm{MHz}$ cavity parameters.

Based upon the 5.4MW of power we wish to couple in

\begin{tabular}{|l|l|}
\hline Radius & 1.2 meters \\
\hline Length & 0.89 meters \\
\hline Electric Field Gradient & $8 \mathrm{MV} / \mathrm{m}$ \\
\hline Power & $5.4 \mathrm{MW}$ \\
\hline Maximum E-field & $31.3 \mathrm{MV} / \mathrm{m} \quad$ (3kilp) \\
\hline Stored Energy & $640 \mathrm{Joules}$ \\
\hline $\mathrm{dF} / \mathrm{dR}$ at outer wall & $-45 \mathrm{kHz} / \mathrm{mm} \mathrm{vol} \mathrm{+}$ \\
\hline Avg. H-field at outer wall & $13900 \mathrm{~A} / \mathrm{m}$ \\
\hline
\end{tabular}

from a $50 \mathrm{ohm}$ coax, we know the coupling loop in the cavity must induce

$$
V_{\text {coax }}=\sqrt{P \times 2 R_{\text {coax }}}=23 \mathrm{kV}
$$

The coupling loop in the cavity must induce this voltage. The loop induces a voltage equal to the integral over the area of the loop of the time derivative of the magnetic field.

$$
\int E \bullet d l=-\int_{S} \frac{\partial B}{\partial t} d S=-j \omega B e^{j \omega t} S
$$

where $\mathrm{B}=\mu \mathrm{H}$ and $\mathrm{S}$ is the area of the loop. From Superfish $\mathrm{H}=13900 \mathrm{~A} / \mathrm{m}$, and so the required induced voltage of 23 $\mathrm{kV}$ can be obtained from a loop of area $\mathrm{S}=0.0029 \mathrm{~m}^{2}$, or roughly a square of $55 \mathrm{~mm}$ per side. The loop would be made $30 \%$ larger in area to allow for matching by rotating the loop to adjust the effective area.

For the tuner a $150 \mathrm{kHz}$ frequency range is required to cover frequency drifts due to temperature variations. A tuner of $300 \mathrm{~mm}$ diameter can accomplish this. The effect of a tuner of this size in a symmetric cavity can be obtained by taking the frequency perturbation value at the wall from Superfish[5] and multiply by the ratio of the area presented by the tuner to the area of the entire $2 \pi$ of cavity wall. For a tuner of $300 \mathrm{~mm}$ diameter a range of $150 \mathrm{~mm}$ meets the requirement.

The coaxial feed line to the cavity must be a minimum of 12 inch coaxial line to avoid breakdown at $23 \mathrm{kV}$ operation. The vacuum window is designed for this same coaxial line dimension.

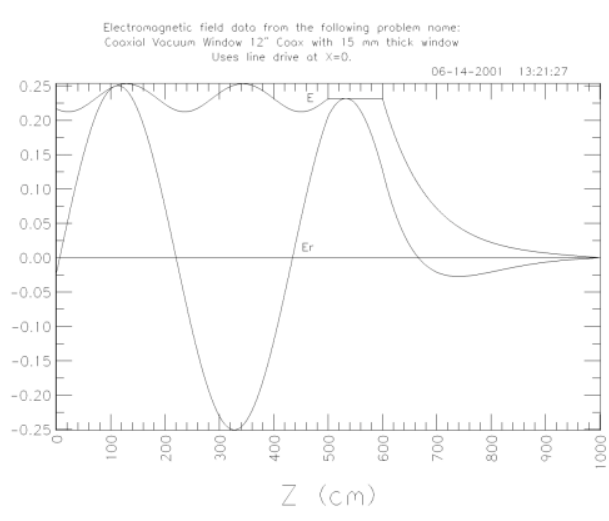

Figure 3. CFISH output plot of the radial component and magnitude of the electric field in a coax with dielectric window and matched load.

The above plot shows the radial component of the electric field $\mathrm{Er}$ and the magnitude of the electric field $\mathrm{E}$ in a coaxial line of $1000 \mathrm{~cm}$ length from the CFISH program[6,7]. A $15 \mathrm{~mm}$ thick window of relative dielectric constant $\varepsilon_{\mathrm{r}}=9.0$ is located at $\mathrm{z}=500 \mathrm{~cm}$. A matched load is created at $\mathrm{z}=600 \mathrm{~cm}$ by filling the coaxial line with a lossy material with the impedance of free space. $\left(\varepsilon_{\mathrm{r}}=0.6+\mathrm{j} 0.8, \mu_{\mathrm{r}}=0.6+\mathrm{j} 0.8\right)$. A line source excites the coax from the left. The VSWR can be obtained by the ratio of the maximum to minimum of the magnitude of the electric field. The VSWR is 1.18:1 which corresponds to a $99.7 \%$ power transmission efficiency. To place the window at a voltage minimum of the reflected wave due to the cavity mismatch during the fill transient the window is to be located $n \lambda / 2$ from the coupling loop where $\mathrm{n}=0,1,2 \ldots \ldots$.

The baseline design above was then developed into a practical cavity that could be engineered to withstand the vacuum loading and other mechanical constraints[8]. After several iterations with AES Inc. the following geometry was chosen for further mechanical engineering.

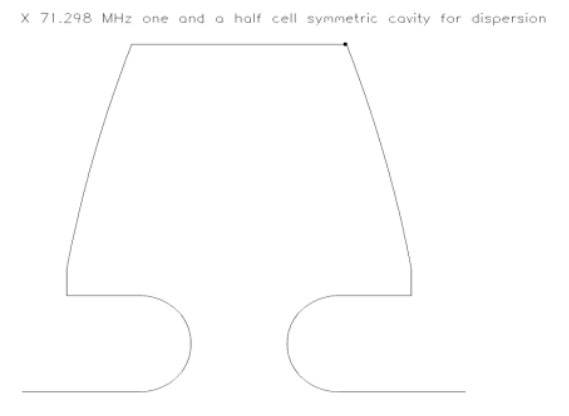

Figure 4. 71.26 MHz cavity with domed sides for mechanical strength. 


\section{CELL TO CELL COUPLING}

The conceptual design for the phase rotation channel requires as high a "real estate" gradient as possible. By this it is meant that the average gradient must include cavity interconnects, magnet focusing regions and drift spaces. This meant packing the cavities as close as possible, and feeding them from separate individually phased power sources. The very large bore tube $(300 \mathrm{~mm}$ radius!) and short interconnects as shown in Figure 1 leads to cell to cell coupling which was investigated with a multicell Superfish analysis. A series of 3 cells with minimum spacing is shown below.

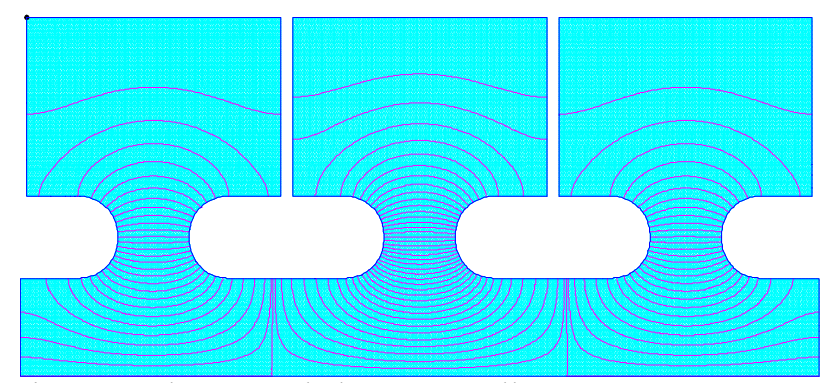

Figure 5. Three coupled $71 \mathrm{MHz}$ cells.

The coupling between cells was found by determining the $0, \pi / 2$, and $\pi$ cavity mode frequencies of the 3 cell structure and then calculate $\kappa$ from the coupling equation:

$$
\omega_{m}^{2}=\omega_{o}^{2}\left\{1-k\left(1-\cos \left(\frac{m \pi}{N}\right)\right)\right\}
$$

where $\omega_{\mathrm{m}}$ is the frequency of the $\mathrm{m}^{\text {th }}$ mode, $\omega_{0}$ the frequency original frequency, $\mathrm{m}$ is the mode number and $\mathrm{N}$ the number of cavities. $\kappa=.00267$, very strongly coupled. For comparison critical coupling between two coupled circuits is calculated as [9]

$$
\kappa=\frac{1}{\sqrt{Q_{1} Q_{2}}}
$$

where the Q's here are $\sim 44000$, or $\kappa_{\mathrm{c}}=.000023$. There is no chance of individual phasing the cavities in this circumstance. Several different cavity spacings were tried and the coupling was not reduced to acceptable levels until the spacing had gone well beyond $\beta \lambda / 2$. At the cavity center to center spacing of $\beta \lambda / 2=1.76$ meters the phase of the cavity drive can be set 180 degrees apart, and the system run with cell to cell coupling. This scheme works, but the average gradient must now include a significant drift space. In the minimum spacing design the center to center spacing is 0.84 meters, and so with the spacing increased to 1.76 meters the distance has doubled, which halves the real estate gradient from $6 \mathrm{MV} / \mathrm{m}$ to $3 \mathrm{MV} / \mathrm{m}$. This is a factor of two lower than what was necessary for the phase rotation channel.

Next, a $30 \mathrm{MHz}$ RF cavity design was looked at to see if operating at the lower frequency would decrease the coupling since the fields would decay faster in the bore hole since the mode is further below cutoff. The coupling for the $30 \mathrm{MHz}$ case was not significantly reduced with $\kappa=0.000237$ with critical coupling $\kappa_{\mathrm{c}}=.000018$, still very strongly coupling. However now spacing at $\beta \lambda / 2$ moves the cavity 4.2 meters center to center, reducing the average gradient in the phase rotation channel to unacceptable levels. In order to reach the original goal of $6 \mathrm{MV} / \mathrm{m}$ average gradient in this frequency range with the $300 \mathrm{~mm}$ bore radius neighboring cavities would have to be electrically shielded from one another with a conductive wall.

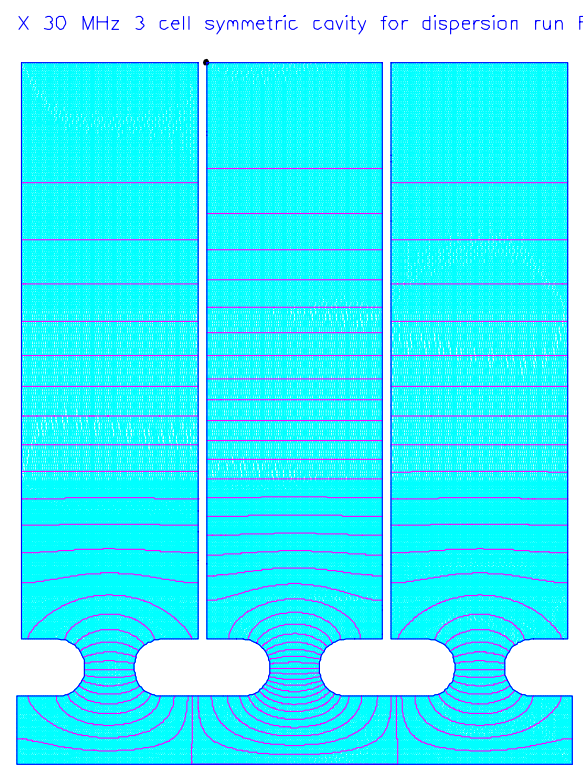

Figure 6. Three coupled $30 \mathrm{MHz}$ cells.

Acknowledgement:The author would like to thank $\mathrm{H}$.

Kirk for his many useful contributions in this work.

\section{REFERENCES}

[1] R. Palmer "Simulation Studies for a High Energy Muon Collider" Presentation at Physics Potential and Development of Muon Colliders, Sausalito, CA, Nov. 16-19 1994

[2] H. Kirk, J.Gallardo, R. Palmer, M. Green, L. Reginato, S.Yu, D. Kaplan, Y. Fuku "Phase Rotation at the Front End of a Neutrino Factory" These proceedings.

[3] R. Weggel, Private communication

[4] J. Corlett, RF working group video conference, http://pubweb.bnl.gov/users/kirk/www/rf_video/aug_12_99/jcorl ett.pdf

[5] K. Halback, R.F. Holsinger, "SUPERFISH-A Computer Program for the Evaluation of RF Cavities with Cylindrical Symmetry", Particle Accelerators 7 (4) (1976)

[6] M. deJong, F.P.Adams "Computation of RF Fields for Accelerator Design", Journal of Microwave and Electromagnetic Energy 27, No. 3,136 (1992)

[7] L.Young, Poisson Code Documentation

[8] J.Rathke, T.Schultheiss, J.Rose, H.Kirk "Conceptual Engineering Design of a Bunch Rotation Cavity for a Pion Production and Capture Experiment" These proceedings [9] F.E.Terman, "Radio Engineers Handbook" McGraw Hill, 1943 